\title{
BMJ Global Health Estimating the burden of leptospirosis: global lessons from Sri Lanka
}

\author{
Janith Warnasekara (D), Shalka Madushan Srimantha, \\ Suneth Buddhika Agampodi
}

To cite: Warnasekara J, Srimantha SM, Agampodi SB. Estimating the burden of leptospirosis: global lessons from Sri Lanka. BMJ Global Health 2021;6:e006946. doi:10.1136/ bmjgh-2021-006946

Handling editor Seye Abimbola

Received 19 July 2021

Accepted 8 October 2021
Check for updates

\section{(C) Author(s) (or their} employer(s)) 2021. Re-use permitted under CC BY-NC. No commercial re-use. See rights and permissions. Published by BMJ

Department of Community Medicine, Faculty of Medicine and Allied Sciences, Rajarata University of Sri Lanka, Anuradhapura, Sri Lanka

Correspondence to Dr Janith Warnasekara; jwarnasekara@yahoo.com

\section{INTRODUCTION}

Health planning requires a comprehensive understanding of the health needs of a population. ${ }^{1}$ Assessments of the actual burden of diseases, including mortality, morbidity and major risk factors, should be a primary concern for decision-making and implementing health policies in the countries. ${ }^{1}$ As a result, assessing the actual burden of many diseases, including leptospirosis, remains a challenge, especially in lower and middle-income countries (LMIC). ${ }^{2}$ The published global disease burden of leptospirosis suffers from a lack of data in various geographical regions and does not have age and sex-disaggregated data. ${ }^{3}$ In addition, the majority of these studies have sampling bias, leading to an underestimation of the true disease burden. Therefore, we discuss the different elements that need to be addressed to properly estimate the burden of leptospirosis using Sri Lanka as an example.

\section{MAIN ELEMENTS IN DISEASE UNDERESTIMATION}

Disease burden estimates heavily rely on routine reporting. Hospital information systems are not digitalised in many LMICs. Thus, routine reporting is a tedious manual procedure that is a 'burden' to treating physicians. Deficiencies in or incomplete reporting/notification represent a significant underlying factor for underestimation. ${ }^{4}$ Reporting is sometimes hampered because the available diagnostic tools are neither perfect nor effective for the early diagnosis of leptospirosis. ${ }^{5}{ }^{6}$ In addition, the clinical features of leptospirosis vary from asymptomatic infection to fatal disease, with the majority of patients having non-specific symptoms. ${ }^{4}$ As a result, on top of not having diagnostic tools, the available imperfect clinical case definitions also lead to misdiagnosis by physicians. ${ }^{4}$ Thus, under notification, lack of diagnostic tools and diverse clinical features could be listed as the three main reasons for
Summary box

Disease underestimation is a primary concern in the field of healthcare globally.

- Leptospirosis is considered an underestimated disease despite being one of the globally widespread zoonotic diseases.

- Publications which mention underestimation as a primary limitation for disease estimation are scarce, especially in lower and middle-income countries including Sri Lanka.

Improving disease notification systems in all health sectors is the first step for accurate estimation of clinically detected cases.

- Developing regionally validated point-of-care diagnostics and describing the clinical profile including uncommon features are key in recognising unidentified cases.

- Accurate prediction of the non-seasonal outbreaks could enhance clinical suspicion of leptospirosis by the treating physicians.

underestimating leptospirosis disease burden (figure 1).

\section{LAPSES IN THE ROUTINE NOTIFICATION}

Notification is the first step in any disease surveillance system. In Sri Lanka, disease notification is a mandatory procedure in which leptospirosis is listed as a notifiable disease. The first contact healthcare provider must fill out a notification form on initial suspicion. Then, the notification form must be sent to the epidemiology unit through the medical officer of health in the area where the patient is residing. The initial part of this process is paper based, as it still happens in many LMICs. However, after the field investigations, the summary report is sent to the epidemiology unit through an electronic database. The notification is a legal requirement for all public, private, complementary and alternative healthcare institutions. However, this ideal procedure does not take place in Sri Lanka. $^{7}$ 


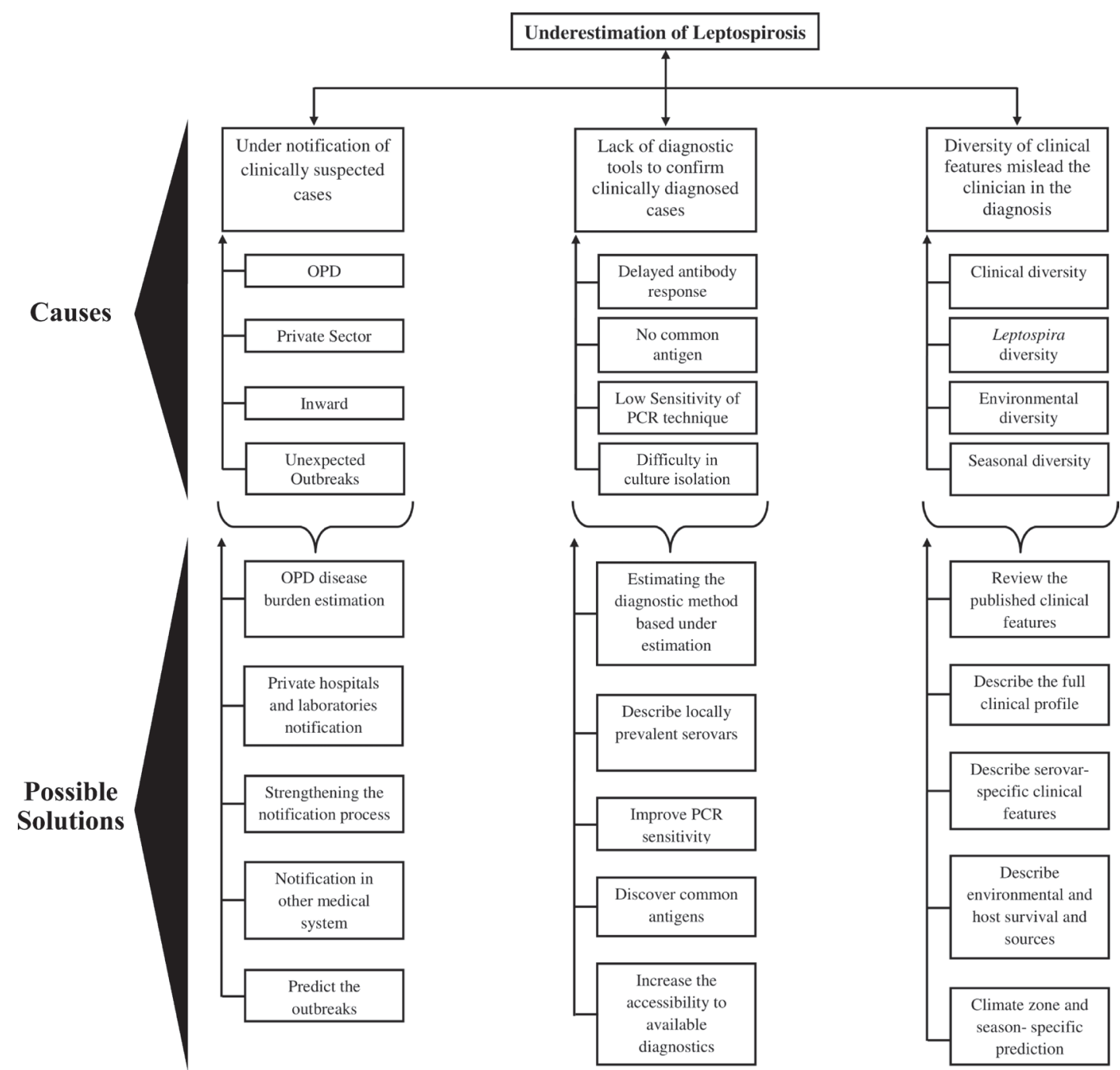

Figure 1 Reasons for the underestimation of leptospirosis and possible actions to improve disease burden estimates

A major limitation of the notification system is the below par reporting from the private sector, where $50 \%$ of ambulatory care services are provided. Despite the availability of free public health services, which include free consultation, laboratory testing and treatment, people prefer the private sector for numerous reasons. Many mild cases of leptospirosis are also managed in the private sector, and they go unreported due to the unavailability of notification forms there. This leads to an eventual underestimation. As a standard protocol, even the private sector laboratories should notify the patients with confirmed cases of infectious diseases. But it has been revealed that only a few laboratories are carrying out this practice. ${ }^{7}$ In addition, a considerable proportion of patients use complementary medicine systems. Notification from complementary medicine also does not occur primarily due to differences in diagnosis. ${ }^{7}$

In public allopathic hospitals, a considerable number of patients are being treated by the outpatient department, and the reporting there is almost zero. ${ }^{8}$ Moreover, the number of leptospirosis cases reported by the notification system shows that the numbers are far less than the numbers documented in the indoor morbidity and mortality report as hospital admissions. ${ }^{4}$ This indicates that despite correct diagnoses, the notification process for hospitalised patients is incomplete. These lapses in notification could be more frequent during non-seasonal and unexpected outbreaks as leptospirosis is mainly associated with monsoon rain. ${ }^{9}$ With the challenge of inadequate investigation facilities, physicians are often left with clinical, biochemical and epidemiological parameters to make a diagnosis. For example, whether the patient worked in a paddy farm is a routine question. At the same time, exposure to minor floodwater or rainwater collection is not considered an epidemiological parameter to define leptospirosis on consultation. Hence, they miss the epidemiological definition for leptospirosis. In addition, outbreaks cause a staggering workload in a demanding and overworked ward. The notification process can be easily missed in such a circumstance as priority is given to critically ill patients. ${ }^{4}$

\section{LACK OF DIAGNOSTIC TOOLS}

Despite all the advances in diagnostic techniques for leptospirosis, ideal, affordable and user-friendly 
diagnostic tests have yet to be discovered..$^{5}$ Diagnostic tests for leptospirosis are categorised into four major groups: culture, direct visualisation through a dark field microscope, molecular (DNA, RNA) or antigen detection and antibody detection. ${ }^{5}$ Direct visualisation and molecular or antigen detection can be performed as early diagnostic techniques, while antibodies are developed a few days after disease onset. Leptospira culture is not a clinically useful diagnostic test as it takes weeks or months to grow. Direct visualisation is also a cumbersome procedure and has an extremely low sensitivity. ${ }^{5}$ Molecular techniques such as quantitative PCR, which are widely used, also have issues due to the high genetic diversity of Leptospira. ${ }^{510}$ Antibody detection techniques in leptospirosis are often not highly useful due to delays in antibody response. ${ }^{5}$ The microscopic agglutination test, which has been considered the gold standard for a long time, has many drawbacks, and clinical utility during the acute phase is limited. ${ }^{6}$ Since none of the available diagnostic methods are ideal, leptospirosis diagnosis is mostly based on clinical, biochemical and epidemiological parameters. This particular problem is not unique to Sri Lanka but a global issue. In Sri Lanka, both the microscopic agglutination test and the PCR tests are available at the Medical Research Institute in Colombo (the commercial capital of Sri Lanka). Two or three other research centres also provide these diagnostic techniques on request. However, clinicians outside Colombo rarely use these facilities due to delays in obtaining results and difficulties in transferring samples. Accordingly, the lack of definitive tests with high clinical utility and the unavailability of tests for clinicians outside the capital prevent the confirmation of cases, contributing to the gross underestimation of leptospirosis.

\section{DIVERSITY OF CLINICAL FEATURES}

Clinical features play a crucial role in diagnosing leptospirosis in places where there are no definitive diagnostic tests available. The clinical presentation of leptospirosis can vary from mild flu-like illness to a disease with multisystemic involvement, and atypical manifestations are not uncommon. ${ }^{4}$ The diversity of clinical features and diseases that mimic leptospirosis, such as hantavirus and dengue infections, can mislead the clinician in the diagnosis. ${ }^{11}$ In addition, the occurrence of several concurrent disease outbreaks, common in the tropics, is a major challenge in diagnosis. This is sometimes complicated by coinfection on a large scale. ${ }^{12}$

Animal studies have shown that the diversity of clinical features of leptospirosis is partially due to different serogroups of Leptospira. ${ }^{13}$ Although the studies are limited, available evidence indicates a similar pattern among humans. ${ }^{14}$ Serogroups are predominantly reservoir specific, and environmental survival depends on diverse factors in different geographical regions. ${ }^{14}$ While the seasonal variation in leptospirosis is well known, ${ }^{9}$ the differences in leptospirosis by climatic zones within countries need further exploration, especially with the observed macrogeographical changes in leptospirosis.

\section{PREDICTING LEPTOSPIROSIS}

Until a definitive diagnostic method is established, correct prediction is the only alternative for detecting the disease. Several mathematical models, such as Faine's scores, have overcome the difficulties in predicting leptospirosis. Some models were used to predict severe leptospirosis using clinical features, and some identified seasonal variations. ${ }^{915}$ However, the clinical utility of these models is questionable. When the number of parameters in the model is higher, the models become complex and incomprehensible. Simplified models incorporating clinical, biochemical and epidemiological parameters will minimise complexities in diagnosing leptospirosis in LMICs. In addition, continuous review of published clinical profiles and studies to describe the complete clinical profile will minimise leptospirosis misdiagnoses.

\section{THE WAY FORWARD}

Most of the shortcomings and challenges we discussed (figure 1) are common to many resource-limited LMICs. However, these limitations were not systematically discussed previously in the literature. Based on the Sri Lankan case scenario, we have identified several main areas for improvement.

Digitalising the surveillance system instead of the traditional postal-based system while strengthening the notification process in all health facilities is fundamental for estimating the disease burden, while strengthening the system, adjusting the estimates for the lack of diagnostic tools and under-reporting from hospitalised patients can be done using mathematical models.

Culture isolation of local serovars and/or cultureindependent identification of subspecies-level Leptospira, improvements and optimisations of diagnostics and the development of new biomarkers are essential in improving the diagnosis. In addition, the clinical spectrum of patients and serogroup-specific clinical syndromes need to be explored to improve the accuracy of clinical diagnosis. This could be supplemented by describing the disease distribution, if possible, with infecting serovars/serogroups. Nevertheless, until achieving the above objectives, adequate funding should be allocated for research development and to improve the accessibility to available diagnostic tests while maintaining universal health coverage.

\section{CONCLUSION}

Systematic approach involving multiple sectors is essential for leptospirosis disease burden estimation in LMICs.

Acknowledgements We thank Dr. Gihan Rathnayaka for support given for graphic designing and Dr. Ishanka Amerasinghe for language editing.

Contributors All three authors were involved in literature search, data curation and manuscript writing. 
Funding SBA and JW are partially supported by the US Public Service Grant (U19Al115658)

Competing interests All Authors declare that there is no competing interest. Patient consent for publication Not required.

Provenance and peer review Not commissioned; externally peer reviewed.

Data availability statement There are no data in this work.

Open access This is an open access article distributed in accordance with the Creative Commons Attribution Non Commercial (CC BY-NC 4.0) license, which permits others to distribute, remix, adapt, build upon this work non-commercially, and license their derivative works on different terms, provided the original work is properly cited, appropriate credit is given, any changes made indicated, and the use is non-commercial. See: http://creativecommons.org/licenses/by-nc/4.0/.

\section{ORCID iDs}

Janith Warnasekara http://orcid.org/0000-0002-6554-6851

Suneth Buddhika Agampodi http://orcid.org/0000-0001-7810-1774

\section{REFERENCES}

1 Lopez AD, Mathers CD. Measuring the global burden of disease and epidemiological transitions: 2002-2030. Ann Trop Med Parasitol 2006;100:481-99.

2 Abela-Ridder B, Sikkema R, Hartskeerl RA. Estimating the burden of human leptospirosis. Int J Antimicrob Agents 2010;36 Suppl 1:S5-7.

3 Costa F, Hagan JE, Calcagno J, et al. Global morbidity and mortality of leptospirosis: a systematic review. PLoS Negl Trop Dis 2015;9:e0003898.

4 Warnasekara J, Koralegedara I, Agampodi S. Estimating the burden of leptospirosis in Sri Lanka; a systematic review. BMC Infect Dis 2019;19:119.

5 Rajapakse S, Rodrigo C, Handunnetti SM, et al. Current immunological and molecular tools for leptospirosis: diagnostics, vaccine design, and biomarkers for predicting severity. Ann Clin Microbiol Antimicrob 2015;14:2

6 Agampodi SB, Dahanayaka NJ, Nöckler K, et al. Redefining gold standard testing for diagnosing leptospirosis: further evidence from a well-characterized, Flood-Related outbreak in Sri Lanka. Am J Trop Med Hyg 2016;95:531-6.

7 Chandrasekar K, Mahesan S, Bath PA, Lecturer S. Notifiable disease surveillance in Sri Lanka and the United Kingdom: a comparative study. Sri Lanka J Bio-Medical Informatics 2013;4:14-22.

8 Warnasekara J, Aberathna P, Nanayakkara G, et al. Improving the leptospirosis disease burden assessment by including ambulatory patients from outpatient departments: a cross-sectional study. F1000Res 2020:9:1129.

9 Warnasekara J, Agampodi S, Abeynayake R R. Time series models for prediction of leptospirosis in different climate zones in Sri Lanka. PLoS One 2021;16:e0248032.

10 Agampodi SB, Matthias MA, Moreno AC, et al. Utility of quantitative polymerase chain reaction in leptospirosis diagnosis: association of level of leptospiremia and clinical manifestations in Sri Lanka. Clin Infect Dis 2012;54:1249-55.

11 Dahanayaka NJ, Agampodi SB, Bandaranayaka AK, et al. Hantavirus infection mimicking leptospirosis: how long are we going to rely on clinical suspicion? J Infect Dev Ctries 2014;8:1072-5.

12 Sunil-Chandra NP, Premaratna R. Evidence of Leptospira and Hanta virus co-infections amongst patients hospitalised for leptospirosislike illness, 2003. Available: http://repository.kln.ac.lk/handle/ 123456789/9365 [Accessed 28 Oct 2017].

13 Goldstein RE, Lin RC, Langston CE, et al. Influence of infecting serogroup on clinical features of leptospirosis in dogs. J Vet Intern Med 2006;20:489-94.

14 Jayasundara D, Senavirathna I, Warnasekara J, et al. 12 nove clonal groups of Leptospira infecting humans in multiple contrasting epidemiological contexts in Sri Lanka. PLoS Negl Trop Dis 2021:15:e0009272.

15 Weeratunga P, Rajapakse S, Niloofa R. A diagnostic scoring model for leptospirosis in resource limited settings, 2016. Available: http:// repository.kln.ac.Ik/handle/123456789/13717 [Accessed 28 Oct 2017] 